\title{
Intuitive and Empirical Prototypes in Childhood Psychopathology
}

\author{
Nicole P. C. M. Krol, Eric E. J. De Bruyn, and John H. L. Van Den Bercken \\ Nijmegen Institute for Cognition and Information, University of Nijmegen
}

\begin{abstract}
This study examines the validity of intuitive classifications of experienced diagnosticians, that is, the empirical foundation of their intuitive prototypes. Intuitive prototypes of 10 experienced diagnosticians were collected in two tasks and were compared with the empirically based assessment system of T. M. Achenbach ( T. M. Achenbach, K. C. Conners, H. C. Quay, F. C. Verhulst, \& C. T. Howell, 1989 ). Results show that the intuitive prototypes correspond very poorly with the empirical core syndromes, instead consisting of different groups of symptoms from various core syndromes. The study also showed a low similarity between behaviors that clinicians judge as co-occurring with an incompletely specified syndrome and behaviors that empirically co-occur with that syndrome. Reasons for the difference between intuitive prototypes and empirically derived classification structures are discussed, as are consequences for diagnostic practice.
\end{abstract}

Research on intuitive prototypes in psychopathology has demonstrated that the prototype view corresponds to how clinicians actually think about and use diagnostic categories (Cantor, Smith, French, \& Mezzich, 1980). Prototypes can be regarded as classes or categories in which features are organized. In the prototype view of classification, category membership is a matter of degree. In the domain of child psychopathology this means that problem behaviors can be more or less typical for a particular prototype. According to Chan and Jackson (1982), intuitive prototypes or implicit personality theories arise from the observation of co-occurrences of behaviors. Therefore, they reflect the distribution of traits in people and provide a relatively valid foundation for the judgement of specific targets.

The issue of whether these intuitive prototypes reflect reality has been raised (Clark, McEwen, Collard, \& Hickok, 1993; Horowitz, Wright, Lowenstein, \& Parad 1981; Rosch, Mervis, Gray, Johnson, \& Boyes-Bream, 1976). However, we are not aware of any study that actually compares the intuitive prototypes of diagnosticians with classes of dysfunctional behavior or syndromes derived from multivariate analyses of systematically collected problem behaviors. The expectation that these intuitive prototypes do not reflect reality is based on various arguments, such as the illusory correlation bias (i.e., seeing a correlation between two events as being stronger than it actually is;

Nicole P. C. M. Krol, Eric E. J. De Bruyn, and John H. L. Van Den Bercken, Department of Special Education, Nijmegen Institute for Cognition and Information, University of Nijmegen, The Netherlands.

We gratefully acknowledge the assistance of Elly Geeraats and Francoise Vermeulen in the collection and analysis of the data. Appreciation is also expressed to Lee R. Beach for comments on an earlier version of this article.

Correspondence concerning this article should be addressed to Nicole P. C. M. Krol, Department of Special Education, Nijmegen Institute for Cognition and Information, University of Nijmegen, P.O. Box 9104 , 6500 HE Nijmegen, The Netherlands. Electronic mail may be sent via Internet to u213312@vm.uci.kun.nl.
Chapman \& Chapman, 1982) or the acknowledged unreliability of clinical judgement.

The present study addresses the empirical anchoring of intuitive prototypes used by individual clinicians. The clinician is viewed as an observer of individual cases-admittedly, a fallible observer. It is assumed that observational experience has created a cognitive structure in the memory of the clinician that includes impressions of the frequency of co-occurrences of problem behaviors. If two behaviors or symptoms are assumed to co-occur frequently, then when one is present, the other is expected. These cognitive structures can be seen as intuitive prototypes (i.e., as categories or classes implicit to the classification knowledge of the clinician). This article questions whether these intuitive prototypes resemble empirical prototypes, that is, syndromes that have been identified empirically by multivariate analyses of systematically collected problem behaviors. This study used the empirical prototypes or core syndromes provided by Achenbach's empirically based Child Behavior Checklist (CBCL) assessment system (Achenbach, Conners, Quay, Verhulst, \& Howell, 1989).

For this study, tasks were developed that presumably involved the intuitive prototypes of clinicians, in particular the knowledge that clinicians have about the co-occurrence of problem behaviors. These tasks were based on a method that is commonly used in basic research on prototypes (Rosch et al., 1976) and in research on psychopathological prototypes (Cantor et al., 1980; Chan \& Jackson, 1982; Horowitz et al., 1981), that is, the method of presenting stimuli and listing associated features or attributes.

\footnotetext{
${ }^{1}$ We used the core syndromes derived from the CBCL because they constitute very solid empirical knowledge that best reflects the reality of co-occurrences of problem behaviors. We did not opt for the categories of the third edition, revised, of the Diagnostic and Statistical Manual of Mental Disorders (DSM-III-R; American Psychiatric Association, 1987 ) as a standard for comparison because it has repeatedly been acknowledged that many of its categories, especially for child psychopathology, lack empirical evidence (DSM-IV Work Group, 1989; Rutter \& Gould, 1985).
} 
Table 1

Description of the Diagnosticians

\begin{tabular}{|c|c|c|c|c|}
\hline Diagnostician & Work setting & Gender & $\begin{array}{l}\text { Experience with } \\
\text { children (years) }\end{array}$ & $\begin{array}{c}\text { Familiar with } \\
\text { CBCL }\end{array}$ \\
\hline 1 & Medical child inpatient clinic & Female & 9 & No \\
\hline 2 & Medical child inpatient clinic & Male & 5 & No \\
\hline 3 & Day clinic for children with developmental problems & Male & 9 & No \\
\hline 4 & Day clinic for children with developmental problems & Female & 8 & No \\
\hline 5 & Child treatment inpatient clinic & Male & 8 & Yes \\
\hline 6 & Child psychiatric outpatient clinic & Male & 10 & No \\
\hline 7 & Child psychiatric outpatient clinic & Male & 17 & No \\
\hline 8 & Child psychiatric outpatient clinic & Male & 3 & No \\
\hline 9 & Child psychiatric outpatient clinic & Male & 11 & No \\
\hline 10 & Child psychiatric outpatient clinic & Female & 4 & No \\
\hline
\end{tabular}

Note. All diagnosticians were clinical child psychologists with master's degrees in psychology. $\mathrm{CBCL}=\mathrm{Child}$ Behavior Checklist.

The study addressed the following questions: (a) What is the correspondence between an intuitive and an empirical clustering of symptoms? and (b) Do problem behaviors that clinicians think of as co-occurring within an incompletely specified syndrome also co-occur empirically?

\section{Method}

\section{Diagnosticians}

The diagnosticians were 10 professional clinical child psychologists (see Table 1). For practical reasons they were selected from the southern part of The Netherlands. Different work settings were chosen that are representative of the child mental health field. All 10 diagnosticians held master's degrees in psychology, were in clinical practice in The Netherlands, and were familiar with diagnosing behavior problems in children of 6 to 11 years. Their experience ranged from 3 to 17 years $($ median $=$ 8.4). Only Diagnostician 5 was familiar with the CBCL system. This sample mirrors the average diagnostician that works in the Dutch child mental health field.

\section{Tasks}

In the construction of the tasks, the CBCL items and the core syndromes of the empirical classification system developed by Achenbach and Edelbrock (1983) were used. Factor analyses of the CBCL revealed core syndromes, that is, clusters of co-occurring problem behaviors that are found in different age and gender groups (Achenbach et al., 1989²). Both the instrument and the resulting classification are reliable and valid for the Dutch population (Achenbach, Verhulst, Baron, \& Akkerhuis, 1987; Verhulst, 1985).

Task 1: Intuitive clustering of the original CBCL symptoms. In Task 1, diagnosticians were asked to cluster the original items of the $\mathrm{CBCL}$

Yvonne refuses to talk and is shy and timid.

She is unhappy and sad.

Figure 1. Example of a vignette presented to the diagnosticians in Task 2. according to their own points of view. Diagnosticians were first given a set of 112 small cards in random order containing the problem behaviors and child characteristics as used by the CBCL. From this set they could select the behaviors and child characteristics with which they were familiar. Problem behaviors and characteristics they had come across in their practice, but which were not in the CBCL set, could be written on new cards.

Next, they were told that some of the problem behaviors and child characteristics co-occur frequently in patients and others co-occur rarely. The diagnosticians were asked to sort the collected behaviors and characteristics into clusters containing items that, in their own clinical experience, co-occurred frequently (no further quantification of the term frequently was given ). They were allowed to form as many clusters as they wanted and to put the same item into more than one cluster. This task addressed the question of whether intuitive clustering agrees with an empirically established system of clusters as represented in the core syndromes of the $\mathrm{CBCL}$.

Task 2: Supplementing incompletely specified syndromes. In Task 2, diagnosticians were asked to supplement seven incompletely specified core syndromes presented as vignettes. Each vignette contained one randomly chosen third of the symptoms of a core syndrome of the CBCL. To imitate clinical practice, we put a name on the vignette, as illustrated in Figure 1, which contains one third of Core Syndrome 7, Withdrawn. Diagnosticians were given the seven vignettes on separate pieces of paper, one by one, in a random order. They were asked to supplement the case description by reporting which additional problem behaviors they expected to be present in that particular child. This task addressed the question of which behaviors are judged to co-occur with the behaviors listed in an incomplete description of an empirical core syndrome and whether these behaviors are similar to the behaviors that empirically co-occur.

\section{Procedure}

For Task 1, there was a 1-hr time limit, and for Task 2, the limit was 5 min for each case description. Task 1 was always given before Task 2 . Tasks 1 and 2 were administered in one session for most diagnosticians. For two diagnosticians, Task 2 was administered in another session because of time constraints. The sessions with the diagnosticians took place in their own offices and were administered by two assistants.

\footnotetext{
${ }^{2}$ The composition of the core syndromes can be found in Achenbach et al. (1989).
} 


\section{Data Analysis and Results}

\section{Task 1: Intuitive Clustering}

Agreement between the intuitive clusters and the empirical core syndromes was examined in two ways. First, the resemblance of the intuitive cluster to a particular core syndrome was computed. That is, the percentage of symptoms in the intuitive cluster that fell into a core syndrome was calculated. For example. assume that a diagnostician has made a cluster labeled "Neurotic" consisting of 15 CBCL items (see Table 2). The items of this intuitive cluster occur in Core Syndromes 1, 2, and $7 .^{3}$ The percentage of items of the intuitive cluster Neurotic that fall into a core syndrome indicates the resemblance of the intuitive cluster to that core syndrome. As can be seen in Table 3, in this example, $6 \%$ of the items of the intuitive cluster Neurotic fall into Core Syndrome 1, Aggressive; $53 \%$ of the items of the intuitive cluster Neurotic fall into Core Syndrome 2, AnxiousDepressed; $29 \%$ of the items fall into Core Syndrome 7, Withdrawn; and $12 \%$ of the items of the intuitive cluster Neurotic fall into no core syndrome. Thus, the intuitive cluster Neurotic contains items from different core syndromes but has the greatest overlap (resemblance) with the empirical core syndrome Anxious-Depressed. When all items of an intuitive cluster are part of a single core syndrome, the resemblance percentage is $100 \%$. Note that this does not imply that the intuitive cluster is exactly the same as the core syndrome, as core syndromes can still contain more items than the ones present in the intuitive cluster.

Returning to the data of the study, 121 intuitive clusters were compared with the nine empirical core syndromes. Of these, only $22(18 \%)$ had a more than $50 \%$ overlap with an empirical

Table 2

Example of the Composition of the Intuitive Prototype (Cluster) Neurotic and the Occurrence of Its Symptoms (Items) in the Core Syndromes of the $C B C L$

\begin{tabular}{|c|c|c|}
\hline & Intuitive cluster Neurotic & $\begin{array}{l}\text { Occurrence in } \\
\text { core syndrome }\end{array}$ \\
\hline 12. & Complains of loneliness & 2 \\
\hline 26. & $\begin{array}{l}\text { Does not seem to feel guilty after } \\
\text { misbehaving }\end{array}$ & 1 \\
\hline 31. & Fears he or she might think or do & 1 \\
\hline & something bad & 2 \\
\hline 32. & Feels he or she has to be perfect & 2 \\
\hline 35. & Feels worthless or inferior & 2 \\
\hline 45 . & Nervous, high-strung, or tense & 2 \\
\hline 50 & Too fearful or anxious & \\
\hline 52 & Feels too guilty & 2 \\
\hline 69. & Secretive & 7 \\
\hline 71. & $\begin{array}{l}\text { Self-conscious or easily } \\
\text { embarrassed }\end{array}$ & 2,7 \\
\hline 75. & Shy or timid & 7 \\
\hline 89. & Suspicious & \\
\hline 103. & Unhappy, sad, depressed & 2,7 \\
\hline 111. & Withdrawn, does not get & \\
\hline & involved with others & 7 \\
\hline 112 & Worrying & 2 \\
\hline
\end{tabular}

Note. Numbers refer to the items of the Child Behavior Checklist (CBCL).
Table 3

Number of Items of the Intuitive Cluster Neurotic That Fall Into the Core Syndromes

\begin{tabular}{lccr}
\hline \multicolumn{1}{c}{ Core syndrome } & $\begin{array}{c}\text { No. of items of } \\
\text { core syndrome }\end{array}$ & $\begin{array}{c}\text { Intuitive cluster } \\
(N=17)\end{array}$ & $\%$ \\
\hline $\begin{array}{l}\text { Externalizing } \\
\text { 1. Aggressive }\end{array}$ & 19 & 1 & 6 \\
$\quad$ 4. Delinquent & 11 & 0 & 0 \\
$\quad$ 3. Attention problems & 9 & 0 & 0 \\
Mixed & & & \\
$\quad$ 5. Schizoid & 5 & 0 & 0 \\
$\quad$ 6. Somatic complaints & 2 & 0 & 0 \\
$\begin{array}{l}\text { Internalizing } \\
\text { 2. Anxious-depressed }\end{array}$ & 13 & 9 & 53 \\
$\quad$ 7. Withdrawn & 9 & 5 & 29 \\
$\begin{array}{l}\text { Boys only } \\
\text { 8. Socially inept }\end{array}$ & 4 & 0 & 0 \\
Girls only & & & \\
$\quad$ 9. Cruel & & 0 & 0 \\
None & 5 & 2 & 12 \\
\hline
\end{tabular}

core syndrome. Most intuitive clusters contained items from different core syndromes. Parts of the empirical groupings of a core syndrome can be found in the intuitive clusters, where they were combined with parts of other core syndromes.

In Table 4, results are given for each diagnostician. It can be seen that 4 of the 12 clusters made by Diagnostician 2 resembled a core syndrome $50 \%$ or more. None of the clusters made by Diagnosticians 1 and 4 had a more than $50 \%$ overlap with an empirical core syndrome.

The small correspondence between intuitive and empirical clusters raises the question of whether clinical significance can be attached to the intuitive clusters. That is, do the intuitive clusters contain enough items of a core syndrome to warrant clinical status in the empirical classification system of Achenbach \& Edelbrock (1983)? In this system, a case obtains clinical status on a syndrome if its score on that syndrome exceeds a certain threshold. Because there were no profiles available with critical or threshold values for the core syndromes at the time of this study, the threshold values for the CBCL syndromes for boys and girls ages $6-11$ from the 1982 profile were used. For all the core syndromes the minimum number of items necessary to obtain a threshold score on that core syndrome was computed ${ }^{4}$ (see Table 5).

Each intuitive cluster was checked to see if it contained enough items of one empirical core syndrome to be classified as clinically significant. In other words, this procedure addressed the question of whether a diagnostician can uniquely identify one core syndrome using his or her intuitive cluster. Table 5 shows that one third (40) of the intuitive clusters contained

\footnotetext{
${ }^{3}$ Note that in Table 2, two items of the intuitive cluster ( 71 and 103) are found in two core syndromes. In computing the percentages of symptoms that fall into the core syndromes, we counted such items twice, so the total number of items in the intuitive cluster in this example is taken to be 17 .

${ }^{4}$ The procedure for this computation can be found in the extended report (Krol, De Bruyn, \& Van Den Bercken, 1995).
} 
Table 4

Number of Times That the Resemblance Between an Intuitive Cluster and a Core Syndrome Was More Than $50 \%$

\begin{tabular}{crrrrrrrrrrrrrr}
\hline & & \multicolumn{10}{c}{ Core syndrome } \\
\cline { 3 - 10 } Diagn & $n$ & 1 & 4 & 3 & 5 & 6 & 2 & 7 & 8 & 9 & Total & $\%$ \\
\hline 1 & 4 & 0 & 0 & 0 & 0 & 0 & 0 & 0 & 0 & 0 & 0 & 0 \\
2 & 12 & 0 & 1 & 0 & 2 & 0 & 1 & 0 & 0 & 0 & 4 & 33 \\
3 & 11 & 0 & 0 & 0 & 1 & 0 & 0 & 0 & 0 & 0 & 1 & 9 \\
4 & 12 & 0 & 0 & 0 & 0 & 0 & 0 & 0 & 0 & 0 & 0 & 0 \\
5 & 11 & 1 & 0 & 1 & 0 & 0 & 1 & 0 & 0 & 0 & 3 & 27 \\
6 & 16 & 1 & 0 & 0 & 1 & 0 & 0 & 0 & 0 & 0 & 2 & 12 \\
7 & 17 & 1 & 0 & 3 & 0 & 0 & 0 & 0 & 0 & 0 & 4 & 23 \\
8 & 7 & 0 & 0 & 0 & 0 & 0 & 0 & 1 & 0 & 0 & 1 & 14 \\
9 & 9 & 1 & 0 & 0 & 0 & 0 & 0 & 0 & 0 & 0 & 1 & 11 \\
10 & 22 & 0 & 0 & 1 & 0 & 0 & 0 & 0 & 5 & 0 & 6 & 27 \\
Sum & 121 & 4 & 1 & 5 & 4 & 0 & 2 & 1 & 5 & 0 & 22 & 18 \\
\hline
\end{tabular}

Note. $n$ represents the number of intuitive clusters made. Diagn $=$ diagnostician. Core syndromes: $1=$ Aggressive, 2 = Anxious-Depressed, 3 = Attention Problems, 4 = Delinquent, $5=$ Schizoid, 6 = Somatic Complaints, $7=$ Withdrawn, $8=$ Socially Inept (boys only), and $9=$ Cruel (girls only).

enough items of an empirical core syndrome to obtain clinical status. However, note that most diagnosticians made two or three intuitive clusters that identified the same core syndrome. Diagnostician 10 is the most extreme example: 11 of her 22 intuitive clusters ( $50 \%$ ) identify the same core syndrome (8) Socially Inept. Diagnosticians 2, 4, and 5 identified the highest number (i.e., four) of different core syndromes as clinically significant using their intuitive clusters. Diagnostician 4 was able to uniquely identify four different core syndromes. It is remarkable that not one of the intuitive clusters of Diagnostician 4 consisted of more than $50 \%$ of items from a core syndrome. Yet, in the empirical classification system, less than $50 \%$ of the items of a syndrome is enough to fall in the clinical range. No one identified the core syndromes Aggressive, Anxious-Depressed, Cruel.

\section{Task 2: Supplementing Incompletely Specified Syndromes}

For each vignette the number of supplemented behaviors was divided by the total number of items that empirically completed the core syndrome. ${ }^{5}$ Results showed that the percentages that indicated the extent to which the diagnosticians added items that also empirically completed the core syndrome were small. Very few of these percentages ( only 5 out of 70 ) were above $50 \%$.

\section{A Note on the Content Validity of the CBCL}

The main interest of this study was not the content validity of the CBCL. However, the items that were not selected from or added to the CBCL item pool in Task 1 provide additional information on the content validity of the CBCL. The added items especially support the conclusion from earlier research on the content validity of the CBCL, namely, that in psychodiagnostic practice, problem behaviors are reported that are not in the item sample of the CBCL (Krol \& De Bruyn, 1990). The fact that intuitive prototypes contain problem behaviors that are not in the CBCL item sample may have influenced the low correspondence between intuitive and empirical prototypes found in the above-mentioned study.

\section{Discussion}

The results show that both the content and the structure of intuitive prototypes have a low correspondence with the content and structure of empirical prototypes. This study is truly explorative in the sense that our results cannot be compared with other research findings. Therefore it should be seen as a first attempt to validate more thoroughly the intuitive prototypes clinicians rely on so heavily during assessment. Intuitive prototypes were compared to empirically derived prototypes with respect to the number of common items. Further research on intuitive prototypes should be directed to the dimensionality of these prototypes using multidimensional scaling and a more advanced metric to capture goodness of fit. Note, however, that this does not negate the present findings of minimal correspondence between intuitive and empirical prototypes.

These results should be interpreted in light of the restricted and perhaps biased character of the individual clinical samples in comparison with the clinical population sample on which the core syndromes of the CBCL system is based. It is assumed that intuitive prototypes or implicit classification structures are based on the observation of co-occurrences of problem behaviors. Therefore, the clinical population with which a clinician has had experience becomes important in the construction of these prototypes. The population from which the empirical syndromes were derived is probably much larger and more diverse than the clinical population on which any individual clinician has constructed his or her prototypes.

In light of our findings, we believe it is important to inform

\footnotetext{
${ }^{5}$ More detailed information about this procedure and the results are given in the extended report (Krol et al., 1995).
} 
Table 5

Number of Times Diagnosticians Were Able to Identify Core Syndromes as Clinically Significant With Their Intuitive Clusters

\begin{tabular}{cccccccccccccc}
\hline & & \multicolumn{10}{c}{ Core syndrome (threshold score) } & & \\
\cline { 3 - 11 } Diagn & $n$ & $1(10)$ & $4(3)$ & $3(4)$ & $5(2)$ & $6(2)$ & $2(7)$ & $7(3)$ & $8(3)$ & $9(4)$ & Total & $\%$ \\
\hline 1 & 4 & 0 & 0 & 0 & 2 & 0 & 0 & 0 & 0 & 0 & 2 & 50 \\
2 & 12 & 0 & 1 & 1 & 2 & 2 & 0 & 0 & 0 & 0 & 6 & 50 \\
3 & 11 & 0 & 0 & 0 & 0 & 1 & 0 & 2 & 0 & 0 & 3 & 27 \\
4 & 12 & 0 & 1 & 1 & 1 & 0 & 0 & 0 & 1 & 0 & 4 & 33 \\
5 & 11 & 0 & 3 & 1 & 1 & 1 & 0 & 0 & 0 & 0 & 6 & 54 \\
6 & 16 & 0 & 1 & 0 & 1 & 0 & 0 & 2 & 0 & 0 & 4 & 25 \\
7 & 17 & 0 & 1 & 2 & 0 & 0 & 0 & 0 & 0 & 0 & 3 & 18 \\
8 & 7 & 0 & 0 & 0 & 0 & 0 & 0 & 0 & 0 & 0 & 0 & 0 \\
9 & 9 & 0 & 0 & 0 & 0 & 0 & 0 & 1 & 0 & 0 & 1 & 11 \\
10 & 22 & 0 & 0 & 0 & 0 & 0 & 0 & 0 & 11 & 0 & 11 & 50 \\
Sum & 121 & 0 & 7 & 5 & 7 & 4 & 0 & 5 & 12 & 0 & 40 & 33 \\
\hline
\end{tabular}

Note. $n$ represents the number of intuitive clusters made. Diagn $=$ diagnostician. Core syndromes: $1=$ Aggressive, $2=$ Anxious-Depressed, $3=$ Attention Problems, $4=$ Delinquent, $5=$ Schizoid, $6=$ Somatic Complaints, $7=$ Withdrawn, $8=$ Socially Inept (boys only), and $9=$ Cruel (girls only).

and teach practitioners about possible differences between their implicit classification systems and empirical multivariate systems. These differences can be analyzed in terms of the contribution of the three explaining factors: fallibility of the diagnostician as observer, the impact of his or her implicit classification theory, and the bias and restriction of his or her clinical sample. This will stimulate the clinician to profit more from the body of empirical knowledge that is emerging in the field of the classification of behavioral and personality problems.

\section{References}

Achenbach, T. M., Conners, K. C., Quay, H. C., Verhulst, F. C., \& Howell, C. T. (1989). Replication of empirically derived syndromes as a basis for taxonomy of child/adolescent psychopathology. Journal of Abnormal Child Psychopathology, 17, 299-322.

Achenbach, T. M., \& Edelbrock, C. S. (1983). Manual for the Child Behavior Checklist and Revised Behavior Profile. Burlington, VT: University of Vermont, Department of Psychiatry.

Achenbach, T. M., Verhulst, F. C., Baron, G. D., \& Akkerhuis, G. W. (1987). Epidemiological comparison of American and Dutch children: I. Behavior and emotional problems and competencies reported by parents for ages 4 to 16. Journal of the American Academy of Child Psychiatry, 26, 317-326.

American Psychiatric Association. (1987). Diagnostic and statistical manual of mental disorders (3rd ed., rev.). Washington, DC: Author.

Cantor, N., Smith, E. E., French, R. D., \& Mezzich, J. (1980). Psychiatric diagnosis as prototype categorization. Journal of Abnormal Psychology, 89, 181-193.

Chan, D. W., \& Jackson, D. N. (1982). Individual differences in the perception and judgement of psychopathology. Multivariate Behovioral Research, 17, 3-32.
Chapman, L. J., \& Chapman, J. (1982). Test results are what you think they are. In D. Kahneman, P. Slovic, \& A. Tversky (Eds.), Judgement under uncertainty: Heuristics and biases (pp. 239-248). Cambridge: Cambridge University Press.

Clark, L. A., McEwen, J. L., Collard, L. M., \& Hickok, L. G. (1993). Symptoms and traits of personality disorder: Two new methods for their assessment. Psychological Assessment, 5, 81-91.

Diagnostic and Statistical Manual of Mental Disorders Work Group. (1989). Child and adolescent psychiatric disorders in DSM-IV: Issues facing the work group. Journal of the American Academy of Child and Adolescent Psychiatry, 28, 830-835.

Horowitz, L. M., Wright, J. C., Lowenstein, E., \& Parad, H. W. (1981 ). The prototype as a construct in abnormal psychology: 1. Method for deriving prototypes. Journal of Abnormal Psychology, 90, 568-574.

Krol, N. P. C. M., \& De Bruyn, E. E. J. (1990). Validity of the CBCL [Letter to the editor]. Journal of the American Academy of Child and Adolescent Psychiatry, 29, 986-987.

Krol, N. P. C. M., De Bruyn, E. E. J., \& Van Den Bercken, J. H. L. (1995). Intuitive and empirical prototypes in childhood psychopathology. (NICI Rep. No. 95-05). Nijmegen, The Netherlands: University of Nijmegen, Department of Special Education.

Rosch, E., Mervis, C. B., Gray, W. D., Johnson, D. M., \& Boyes-Bream, P. (1976). Basic objects in natural categories. Cognitive Psychology, 8, 382-439.

Rutter, M., \& Gould, M. (1985). Classification. In M. Rutter \& L. Hersov (Eds.), Child and adolescent psychiatry (pp. 304-321). London: Blackwell.

Verhulst, F. C. (1985). Mental health in Dutch children. Meppel: Krips.

Received November 28, 1994

Revision received June 3, 1995

Accepted June 8, 1995 